\title{
Decreased expression of protein tyrosine phosphatase non-receptor type 12 is involved in the proliferation and recurrence of bladder transitional cell carcinoma
}

\author{
YONGRUI PIAO ${ }^{1}$, XIANKUI LIU ${ }^{2}$, ZHENHUA LIN $^{3}$, ZHEHU JIN $^{4}$, \\ XUANSHUN JIN ${ }^{5}$, KUICHANG YUAN ${ }^{5}$ and WENYUAN WU ${ }^{2}$ \\ ${ }^{1}$ Department of Urology, Affiliated Hospital of Yanbian University, Yanji, Jilin 133000; \\ ${ }^{2}$ Department of Urology, The First Hospital of China Medical University, Shenyang, Liaoning 110001; \\ ${ }^{3}$ Department of Pathology, Yanbian University Medical College; ${ }^{4}$ Department of Dermatology and Venereology; \\ ${ }^{5}$ Department of Cardiology, Affiliated Hospital of Yanbian University, Yanji, Jilin 133000, P.R. China
}

Received August 13, 2014; Accepted April 24, 2015

DOI: $10.3892 / \mathrm{ol} .2015 .3454$

\begin{abstract}
Protein tyrosine phosphatase non-receptor type 12 (PTPN12) has been shown to be involved in the development of a number of types of carcinoma. However, the effect of PTPN12 on the proliferation and recurrence of human bladder transitional cell carcinoma (TCC) is unclear. The present study aimed to investigate the expression and function of PTPN12 in human TCC. Samples from 164 patients with TCC, in addition to 146 patients undergoing bladder surgery for indications other than TCC, were examined. PTPN12 protein expression was examined using immunohistochemistry and western blotting, and PTPN12 mRNA expression was examined using reverse transcription-quantitative polymerase chain reaction. PTPN12 expression was increased following transfection with the PTPN12-expressing, pcDEF3 vector, and PTPN12 expression was decreased by RNA interference, in four TCC cell lines. The proliferation of TCC cells was analyzed by a WST-1 assay and in xenografts on BALB/C nude mice. The effect of PTPN12 on tumor recurrence was analyzed by adhesion, migration and invasion assays in TCC cell lines. PTPN12 expression was significantly decreased in TCC tissues compared with that in normal urothelium, and the level of PTPN12 expression was negatively correlated with tumor size, pathological grade, clinical stage and tumor recurrence. Furthermore, decreased expression of PTPN12 significantly enhanced the proliferation of TCC cells in vitro and in vivo. TCC cells with lower levels of PTPN12 exhibited greater adhesion, migration and invasion. In conclusion, PTPN12 expression is downregulated in
\end{abstract}

Correspondence to: Dr Xiankui Liu, Department of Urology, The First Hospital of China Medical University, 155 Nanjing North Road, Heping, Shenyang 110001, P.R. China

E-mail: xiankuiliu78@163.com

Key words: protein tyrosine phosphatase non-receptor type 12, proliferation, bladder transitional cell carcinoma human TCC. Restoring PTPN12 activity may represent a novel therapeutic strategy for this disease.

\section{Introduction}

Bladder urothelial carcinoma is the most common malignant urinary tract tumor worldwide, and is characterized by a high level of morbidity and mortality $(1,2)$. Of bladder tumors, $90 \%$ are transitional cell carcinomas (TCCs) (1). Approximately $80 \%$ of TCCs are non-muscle-invasive (pTa-pT1), while $20 \%$ of TCCs are muscle-invasive (pT2-pT4). Between 10-30\% of non-muscle-invasive TCCs will develop into muscle-invasive TCCs, and $50-70 \%$ of non-muscle-invasive TCCs recur post-operatively (3). The 5-year overall survival rate of non-muscle-invasive TCCs is $90 \%$, while it is $\sim 60 \%$ for pT2 TCCs, $35 \%$ for pT3 TCCs, and $25 \%$ for pT4 TCCs $(4,5)$. The prognosis of TCC primarily depends on the clinical stage and the histological grade at the time of diagnosis (6). Although surveillance strategies and therapeutic options for patients with TCCs have developed in recent years, these advances have not diminished the mortality or recurrence rates of TCCs. In addition, the molecular mechanisms underlying the development and patterns of recurrence of TCCs, require further evaluation. Indeed, the identification of novel molecular targets is necessary in order to devise improved therapeutic approaches.

Dynamic phosphorylation events are involved in signaling transduction, and are tightly regulated by protein kinases and phosphatases. Protein tyrosine phosphatases (PTPs) serve as inhibitors of tyrosine kinase signaling, and are involved in tumor suppression (7). PTPs have also been reported to exhibit functions in cellular physiology and carcinogenesis $(8,9)$. PTP non-receptor type 12 (PTPN12) is a member of the PTP family and is located at 7q11.23 (10). PTPN12 has recently been identified as a tumor suppressor gene, which is associated with progression in human breast cancer (11). Another recent study suggested that decreased expression of PTPN12 leads to the upregulation of HER2/EGFR activity in HER2-negative breast cancer, and that PTPN12 may act as a tumor suppressor gene in HER2-negative breast cancer (12). There is clear 
evidence of reduced expression of PTPN12 in various human tumors, including esophageal squamous cell cancer and colon cancer $(13,14)$. Adhesion, migration and invasion of tumor cells may increase the metastasis of malignant tumors, and are also important factors in tumor recurrence. A number of studies have indicated that PTPN12 is a ubiquitously expressed cytosolic phosphatase, and is a key stimulator of cell migration and invasion $(15,16)$. PTPN12 has also been reported to inhibit multiple oncogenic tyrosine kinases, in addition to mediating the regulation of cell adhesion, migration and metastasis (17). Furthermore, suppressing PTPN12 expression may enhance the migration of colon (14) and ovarian (10) cancer cells, which suggests that PTPN12 may lead to metastasis in human malignancies. The effect of PTPN12 on the recurrence of TCCs is unclear, as TCC is a malignancy with a high recurrence rate. Therefore, the expression of PTPN12 and its function in TCC requires further elucidation.

The current study investigated the expression of PTPN12 and its effect on proliferation and recurrence in human TCC. The results indicated that PTPN12 expression is decreased in TCC, and that decreased PTPN12 expression suppresses the proliferation, adhesion, migration and invasion of TCC cells. Thus, loss of PTPN12 activity appears to be involved in the progression and recurrence of TCC, and PTPN12 may be a suitable biomarker for TCC recurrence. Furthermore, the present findings also suggested that restoring PTPN12 activity may represent a therapeutic target for TCC. The current study investigated the expression of PTPN12 and its effect on proliferation and recurrence in human TCC. PTPN12 may be a suitable biomarker in targeting TCC to prevent recurrence.

\section{Materials and methods}

Patients and samples. This study was approved by the Ethics Committee of Affiliated Hospital of YanBian University Hospital (Yanji, China). One hundred and sixty-four patients with TCC, who were treated in the Department of Urology between 2008 and 2013 were randomly selected. No patients with TCC received chemotherapy or radiotherapy pre-operatively. Determination of the histological cell type of the resected bladder cancer samples was conducted by an experienced pathologist. All of the tumors in the present study were from conventional TCCs. In addition, normal urothelial samples were obtained from 146 patients who underwent bladder surgery due to diseases other than TCC, such as urinary trauma and benign prostatic hyperplasia. The histological grade was evaluated according to the World Health Organization (WHO) 2004 grading system for TCC (18). The tumor stage was also assessed according to the Union for International Cancer Control (UICC) 2009 TNM classification system (www.uicc.org/resources/tnm). All TCC and normal bladder tissues were formalin-fixed and paraffin-embedded. Furthermore, all samples in the current study were immediately frozen in liquid nitrogen following resection, and then maintained at $-90^{\circ} \mathrm{C}$ prior to protein and total RNA extraction. Written informed consent was obtained from all patients prior to the study.

Cell culture. Four TCC cell lines, 253 J, T24, RT112 and TCCSUP, were used in the current study. All cell lines were purchased from the American Type Culture Collection (Manassas, VA, USA). TCC cells were cultured with complete medium consisting of RPMI-1640 medium (Gibco, Glasgow, Scotland) with $10 \%$ heat-inactivated fetal bovine serum, and supplemented with $2 \mathrm{mM}$ L-glutamine, $25 \mathrm{mM}$ HEPES, $1 \%$ non-essential amino acids, $100 \mu \mathrm{g} / \mathrm{ml}$ streptomycin, and 100 units/ml penicillin (All from Sigma-Aldrich, St. Louis, MO, USA). All TCC cell lines were incubated as monolayers in a $10-\mathrm{cm}$ plastic dish and cultured in a humidified atmosphere at $37^{\circ} \mathrm{C}$ with $5 \% \mathrm{CO}_{2}$.

Immunohistochemistry. Paraffin slices $(4-\mu \mathrm{m})$ were deparaffinized in xylene and then rehydrated with graded alcohol. Endogenous peroxidase activity was suppressed with $0.3 \%$ hydrogen peroxide for $30 \mathrm{~min}$. All slices were also blocked with $20 \%$ rabbit serum for 20 min prior to $3 \mathrm{~h}$ of incubation with the appropriate primary antibody. Subsequently, all slices were incubated with a polyclonal rabbit anti-human PTPN12 antibody (1:500 dilution; ab90641) or monoclonal mouse anti-human $\beta$-actin (1:5,000 dilution; ab6276) purchased from Abcam (Cambridge, MA, USA) at $4^{\circ} \mathrm{C}$ for $16 \mathrm{~h}$. The slices were washed three times with Tris-buffered saline and incubated with biotinylated goat anti-rabbit immunoglobulins $(1: 3,000$ dilution; E0432; DAKO, Glostrup, Denmark) at $4^{\circ} \mathrm{C}$ for $16 \mathrm{~h}$. Antigen retrieval was conducted by microwave heating of tissue sections attached to microscope slides to temperatures up to $100^{\circ} \mathrm{C}$. Determination of the antibody reaction was conducted with a streptavidin-biotin complex. The results of the immunohistochemical analysis were examined using a CX31 binocular light microscope (Olympus Corporation, Tokyo, Japan). PTPN12 immunostaining was semi-quantitatively assessed for positive intensity using the following grades: -, negative; +, weak; ++, moderate; and +++, strong.

Reverse transcription-quantitative polymerase chain reaction $(R T-q P C R)$. Total RNA was extracted from TCC and normal bladder samples with a Quick PrepmRNA purification kit (GE Healthcare, Buckinghamshire, UK), according to the manufacturer's instructions. The first-strand cDNA was then produced, using a synthesis kit (Amersham Biosciences, Little Chalfont, UK) for reverse transcription. RT-PCR was also performed according to the manufacturer's instructions. PCR products were evaluated using agarose gel electrophoresis. qPCR was performed using LC FastStart DNA Master SYBR Green I (Roche, Indianapolis, IN, USA). The cycling conditions for the PCR were as follows: Denaturation $\left(95^{\circ} \mathrm{C}\right.$ for $5 \mathrm{~min}$ ), annealing ( 30 cycles of $95^{\circ} \mathrm{C}$ for $30 \mathrm{sec}, 60^{\circ} \mathrm{C}$ for $30 \mathrm{sec}$ and $72^{\circ} \mathrm{C}$ for $\left.1 \mathrm{~min}\right)$ and extension $\left(72^{\circ} \mathrm{C}\right.$ for $\left.10 \mathrm{~min}\right)$. The PCR products were then quantified with a Light Cycler (Roche). The primers for human PTPN12 were designed by Primer Premier, version 5.0 (Premier Biosoft International, Toronto, Canada), and the sequences were as follows: Forward: 5'-AATACTGCAGCCACCGGAA-3' and reverse: 5'-CAACACTGGCTTTGGATG-3'. The product size was $124 \mathrm{bp}$. GAPDH was used as an internal control, and the primer sequences were as follows: Forward: 5'-TCAAGAAGGTGGTGAAGCAG-3' and reverse: 5'-GTGGAGGAGTGGGTGTC-3'.

Western blotting. Western blotting was performed according to the manufacturer's instructions. Total protein was extracted 
using lysis buffer and the protein concentration was detected using the Bradford dye-binding protein assay (Bio-Rad Laboratories, Richmond, CA, USA). SDS polyacrylamide gel electrophoresis was then conducted. The rabbit anti-PTPN12 polyclonal antibody was obtained from Abcam, and an anti- $\beta$-actin monoclonal antibody (Abcam) was used as an internal control. The immune complexes were detected using an ECL system (Amersham, Aylesbury, UK).

RNA interference (RNAi) and transfection. All small interfering RNA (siRNA) oligonucleotide sequences for PTPN12 were designed using siDirect software, version 2.0 (http:// sidirect2.rnai.jp/). TCC cells were seeded in $10-\mathrm{cm}$ culture dishes with complete medium (no antibiotics) until they reached $50 \%$ confluence. TCC cells were subsequently transfected with siRNA oligonucleotides (Shanghai Shenggong Biology Engineering Technology Service, Ltd., Shanghai, China) using Lipofectamine $2000^{\circledR}$ (Invitrogen Life Technologies, CA, USA). Following culture for 2 days, the expression of PTPN12 was examined by RT-qPCR. The human cDNA coding sequence of PTPN12 was cloned by RT-PCR, using a normal bladder sample as a substrate. The PCR products were then sub-cloned into a pcDEF3 vector (Sigma-Aldrich). The four TCC cell lines were also stably transfected with a pcDEF3 vector containing the full-length cDNA of PTPN12, using Lipofectamine 2000. Monoclonal TCC cell lines were selected with G418 (Sigma-Aldrich) and the expression of PTPN12 was also measured using RT-qPCR.

Proliferation analysis. The effect of PTPN12 on the proliferative ability of TCC cells was analyzed by a WST-1 assay. Exponentially growing TCC cells $\left(2 \times 10^{3}\right)$ were harvested and seeded into 96-well microtiter plates. Following continuous incubation for 1, 2 or 3 days, $10 \mu \mathrm{l}$ of WST-1 (Roche, Penzberg, Germany) was added to each well and the incubation was continued for a further $2 \mathrm{~h}$. The absorbance of each well, which represented the cell count, was examined using a microculture plate reader (Immunoreader, Tokyo, Japan) at $450 \mathrm{~nm}$.

Animal xenograft experiments. Thirty male BALB/C nude mice (4-6 weeks old; weight, 16-20 g) were maintained under specific pathogen-free conditions at $25^{\circ} \mathrm{C}$ in a relative humidity of $55 \%$, with free access to food and water. The mice were randomly divided into two groups: Control and PTPN12 vector. A total of $4 \times 10^{8}$ TCC cells were injected into the backs of nude mice. The mice were then observed continuously for 5 weeks. The volume of each TCC tumor was measured once per week. After 5 weeks, the mice were sacrificed under deep anesthesia by intraperitoneal injection of pentobarbital sodium $(80 \mathrm{mg} / \mathrm{kg}$ ), and the final volume of each TCC tumor was recorded.

\section{Adhesion, migration and invasion assays}

Adhesion assay. 24-well culture plates were coated with $40 \mu \mathrm{g}$ of Matrigel/well (BD Biosciences, San Jose, CA, USA) and air-dried for $60 \mathrm{~min}$. A total of $6 \times 10^{4} \mathrm{TCC}$ cells were suspended with RPMI-1640, containing $0.6 \%$ bovine serum albumin, and added to each well. TCC cells were incubated for $2 \mathrm{~h}$ and washed three times in order to remove unattached
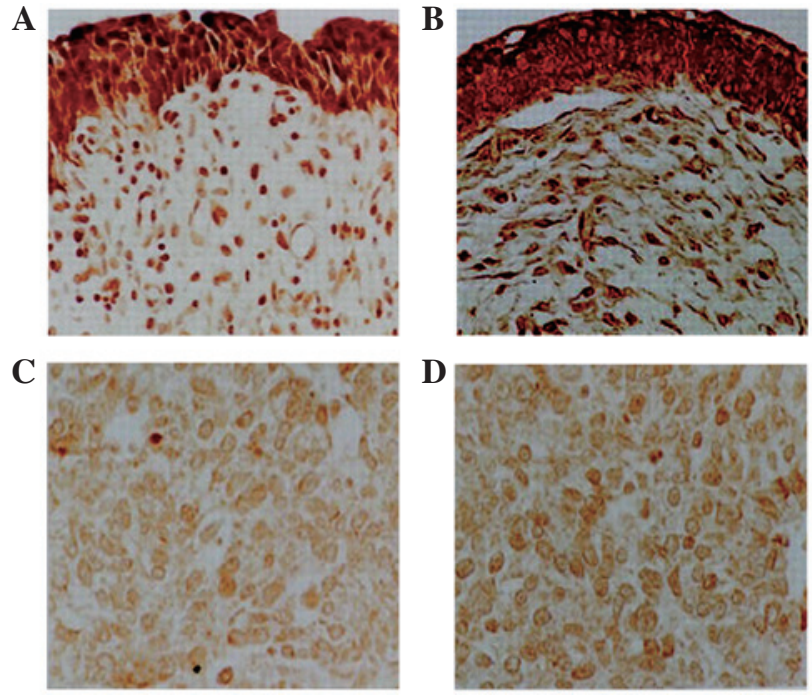

Figure 1. PTPN12 protein expression in two paired bladder tissue samples was analyzed by immunohistochemical staining. Low PTPN12 expression in TCC tissues obtained from stage (A) $\mathrm{T} 2{ }_{\mathrm{a}} \mathrm{N}_{0} \mathrm{M}_{0}$ and (B) $\mathrm{T} 2{ }_{\mathrm{b}} \mathrm{N}_{0} \mathrm{M}_{0}$ patients. High PTPN12 expression in the corresponding normal urothelium tissues of the stage (C) $\mathrm{T} 2{ }_{\mathrm{a}} \mathrm{N}_{0} \mathrm{M}_{0}$ and (D) $\mathrm{T} 2{ }_{\mathrm{b}} \mathrm{N}_{0} \mathrm{M}_{0}$ patients. Magnification, $\mathrm{x} 200$. PTPN12, protein tyrosine phosphatase non-receptor type 12; TCC, transitional cell carcinoma.

cells. The cells attached to the bottom of the culture plate were stained with hematoxylin and counted under a microscope.

Migration assay. The migration assay was performed with a 24-well Transwell system (Poretics Corp., Livermore, CA, USA), which contained $8 \mu \mathrm{m}$-pore polycarbonate membrane filters. TCC cells were seeded into the upper wells at a density of $2 \times 10^{4}$ cells/well and incubated at $37^{\circ} \mathrm{C}$ for $24 \mathrm{~h}$. The polycarbonate membranes were removed, fixed with $70 \%$ ethanol and stained with hematoxylin for $60 \mathrm{~min}$. The upper sides of the membranes were scraped to remove cells that had attached, but not migrated, and the membranes were then mounted onto a microscope slide. Chemotaxis was analyzed in each well by counting the number of TCC cells in 15 randomized fields under a microscope.

Invasion assay. The invasion assay was performed with a Transwell system (Corning, Inc., Corning, NY, USA). The diameter of the filter membrane was $6.5 \mathrm{~mm}$ and the pore size was $8 \mu \mathrm{m}$. Matrigel was added to the filter, forming a thin gel layer, and $2 \times 10^{5}$ TCC cells, suspended with serum-free medium, were seeded into the upper Transwell chamber. Following incubation for $24 \mathrm{~h}$, TCC cells on the upper filter were removed, while cells that had penetrated to the lower filter were stained with hematoxylin. Cells in 15 randomized fields were counted under a microscope.

Statistical analysis. Statistical calculations were performed using SPSS version 16.0 (SPSS, Inc., Chicago, IL, USA). All experiments were conducted in triplicate and the results are presented as the mean \pm standard deviation. Data were analyzed using Student's t-test. A $\chi^{2}$ test was also conducted, in order to assess the correlation between the expression of PTPN12 and the clinical characteristics of patients with TCC. $\mathrm{P}<0.05$ was considered to indicate a statistically significant difference. 
Table I. Association between PTPN12 expression and patient characteristics.

\begin{tabular}{|c|c|c|c|c|c|c|c|c|}
\hline \multirow[b]{2}{*}{ Characteristic } & \multirow[b]{2}{*}{ Number } & \multirow[b]{2}{*}{$\begin{array}{l}\text { PTPN12 mRNA } \\
(\text { mean } \pm \text { SD })\end{array}$} & \multirow[b]{2}{*}{ P-value } & \multicolumn{5}{|c|}{ PTPN12 protein } \\
\hline & & & & $\begin{array}{l}- \\
(\mathrm{n})\end{array}$ & $\begin{array}{l}+ \\
(\mathrm{n})\end{array}$ & $\begin{array}{l}++ \\
(\mathrm{n})\end{array}$ & $\begin{array}{l}+++ \\
(\mathrm{n})\end{array}$ & P-value \\
\hline TCC & 164 & $3.24 \pm 0.36$ & & 24 & 64 & 59 & 17 & \\
\hline NT & 146 & $7.71 \pm 0.61$ & $<0.05$ & 2 & 4 & 74 & 66 & $<0.05$ \\
\hline Gender & & & $>0.05$ & & & & & $>0.05$ \\
\hline Male & 106 & $3.25 \pm 0.39$ & & 16 & 41 & 38 & 11 & \\
\hline Female & 58 & $3.22 \pm 0.37$ & & 8 & 23 & 21 & 6 & \\
\hline Age (years) & & & $>0.05$ & & & & & $>0.05$ \\
\hline$<60$ & 99 & $3.28 \pm 0.36$ & & 15 & 41 & 33 & 10 & \\
\hline$\geq 60$ & 65 & $3.18 \pm 0.33$ & & 9 & 23 & 26 & 7 & \\
\hline Tumor size $(\mathrm{cm})$ & & & $<0.05$ & & & & & $<0.05$ \\
\hline$<3$ & 84 & $4.89 \pm 0.48$ & & 6 & 21 & 45 & 12 & \\
\hline$\geq 3$ & 80 & $1.52 \pm 0.17$ & & 8 & 43 & 14 & 5 & \\
\hline Histologic grade & & & $<0.05$ & & & & & $<0.05$ \\
\hline I & 67 & $4.49 \pm 0.42$ & & 3 & 12 & 38 & 14 & \\
\hline II & 55 & $3.14 \pm 0.32$ & & 5 & 31 & 16 & 3 & \\
\hline III & 42 & $1.37 \pm 0.15$ & & 16 & 21 & 5 & 0 & \\
\hline Clinical stage & & & $<0.05$ & & & & & $<0.05$ \\
\hline $\mathrm{T}_{1}-\mathrm{T}_{2}$ & 88 & $4.58 \pm 0.43$ & & 5 & 23 & 47 & 13 & \\
\hline $\mathrm{T}_{3}-\mathrm{T}_{4}$ & 76 & $1.68 \pm 0.17$ & & 19 & 41 & 12 & 4 & \\
\hline Primary or recurrence & & & $<0.05$ & & & & & $<0.05$ \\
\hline Primary & 86 & $4.69 \pm 0.39$ & & 4 & 20 & 48 & 14 & \\
\hline Recurrence & 78 & $1.56 \pm 0.18$ & & 20 & 44 & 11 & 3 & \\
\hline
\end{tabular}

PTPN12, protein tyrosine phosphatase non-receptor type 12; TCC, transitional cell carcinoma, NT, normal urothelium tissue; RT-qPCR, reverse transcription-quantitative polymerase chain reaction; $\mathrm{SD}$, standard deviation; $\mathrm{n}$, number.

\section{Results}

Patient characteristics. Data from 106 male and 58 female patients were obtained in the current study. Patient ages ranged from 36-88 years (median, 58 years) and tumor size ranged from $0.5-21 \mathrm{~cm}$ (median, $1.5 \mathrm{~cm}$ ). Eighty-six patients were diagnosed with primary TCC disease, and 78 patients had previously been treated with cystectomy or transurethral resection. Of 164 patients with TCC, 67 were classified as grade I, 55 as grade II and 42 as grade III, according to the WHO 2004 grading system for TCC. Clinical stage was evaluated according to the UICC 2009 TNM staging system. The numbers of patients with disease at each stage were as follows: T1, 46; T2, 42; T3, 41; and T4, 25 (Table I). The follow-up period was 25-59 months. Presenting symptoms included abdominal pain (21 patients), hematuria (113 patients), palpable masses (3 patients) and urinary discomfort (48 patients). Fourteen patients were anemic, 6 patients had metastatic TCC and 41 patients had $>2$ concomitant diseases at the time of diagnosis, including angina pectoris, urolithiasis and diabetes mellitus.

IHC analysis of PTPN12 expression in TCC. The expression of PTPN12 protein in normal human urothelium and TCC tissues
A

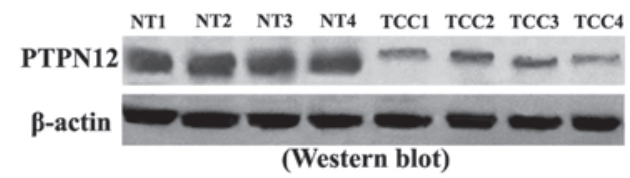

B

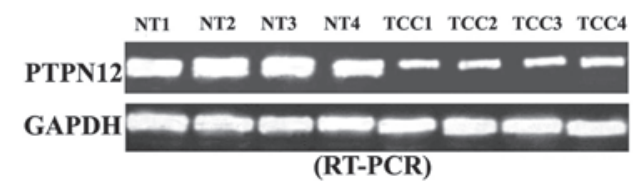

C

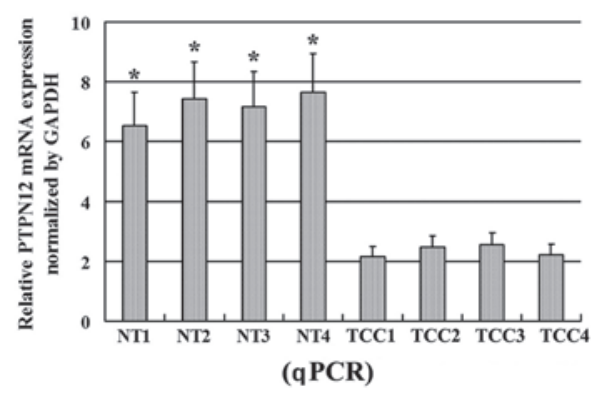

Figure 2. PTPN12 expression was detected by (A) western blotting, (B) RT-PCR and (C) qPCR. All experiments were performed in triplicate. Data are presented as the mean \pm standard deviation. ${ }^{*} \mathrm{P}<0.05$ vs. TCCs. PTPN12, protein tyrosine phosphatase non-receptor type 12 ; TCC, transitional cell carcinoma; NT, normal urothelium tissue; RT-PCR reverse transcription-polymerase chain reaction; qPCR, quantitative PCR. 


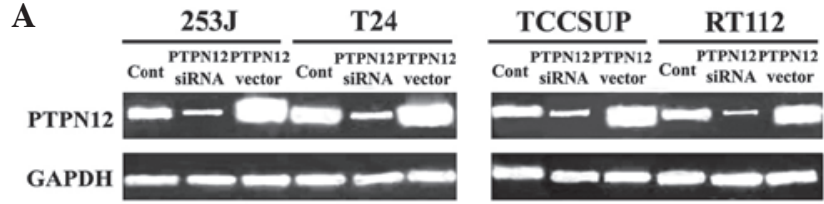

B

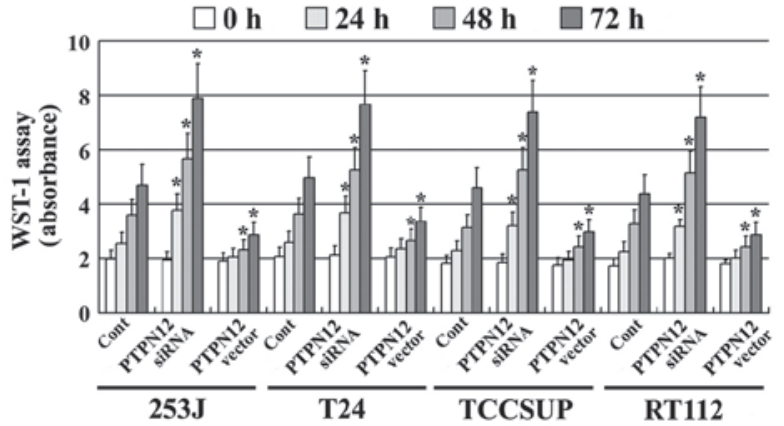

C

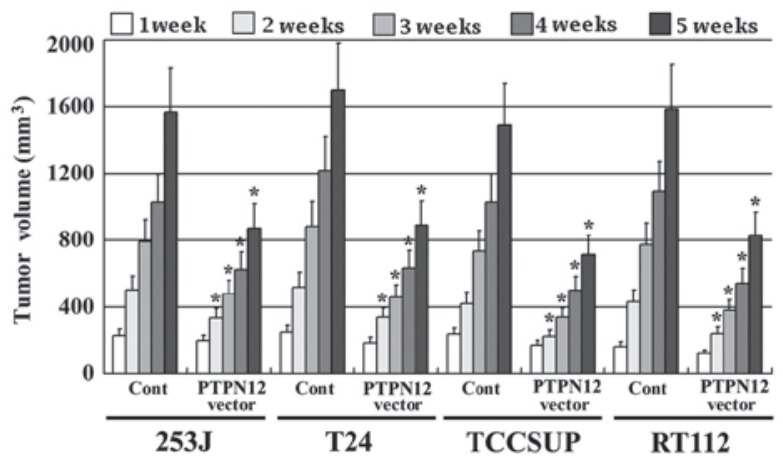

Figure 3. Effect of PTPN12 on the proliferative ability of TCC cells. The pcDEF3 vector of PTPN12 was stably transfected into TCC cell lines PTPN12 expression was also decreased using RNA interference technology. (A) All transfections were confirmed by reverse transcription-quantitative polymerase chain reaction. The proliferation of TCC cells in vitro was examined by (B) WST-1 assay and (C) xenografts on BALB/C nude mice. ${ }^{*} \mathrm{P}<0.05$ vs. cont. PTPN12, protein tyrosine phosphatase non-receptor type 12; TCC, transitional cell carcinoma; siRNA, small interfering RNA; cont, control.

was determined by immunohistochemistry. The expression of PTPN12 in TCC tissue samples was significantly decreased compared with that in normal urothelium (Fig. 1). PTPN12 expression was detected in 140 of 164 TCC specimens (85.4\%). While $46.3 \%$ of TCC specimens exhibited medium or strong expression of PTPN12. By contrast, PTPN12 expression was detected in 144 of 146 normal urothelium tissues (98.6\%) and $94.5 \%$ of TCC exhibited medium or strong PTPN12 expression. With respect to the correlation between clinical characteristics and PTPN12 expression, PTPN12 expression was found to be negatively associated with tumor size, pathological grade, clinical stage and tumor recurrence, when assessed using $\chi^{2}$ analysis. However, gender and age were not correlated with PTPN12 expression (Table I). These findings demonstrated that a reduction in PTPN12 protein expression may be involved in the pathogenesis of human TCC.

RT-qPCR and western blot analysis of PTPN12 expression in TCC. PTPN12 expression in normal human urothelium and TCC tissues was also determined by RT-qPCR and western blotting. The relative level of PTPN12 expression is represented as a ratio to that of the internal control. PTPN12
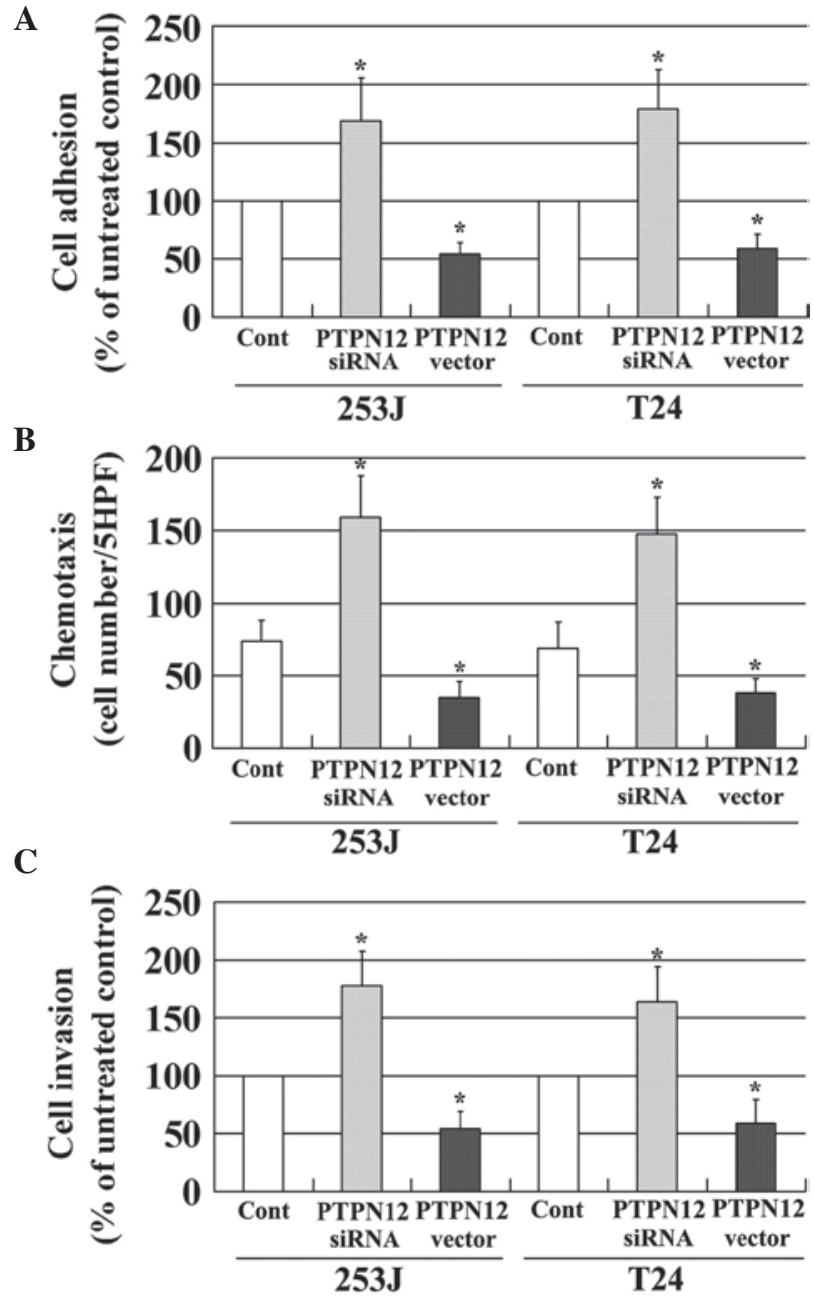

Figure 4. PTPN12 expression was increased by pcDEF3 vector transfection and decreased by siRNA. PTPN12 decreased the (A) adhesion, (B) migration and $(\mathrm{C})$ invasion of TCC cells. ${ }^{*} \mathrm{P}<0.05$ vs. cont. PTPN12, protein tyrosine phosphatase non-receptor type 12; siRNA, small interfering RNA; TCC, transitional cell carcinoma; cont, control.

expression was significantly downregulated in TCC tissues compared with that in normal urothelium tissues, and PTPN12 expression was similar to the levels detected using immunohistochemistry. In addition, PTPN12 expression in TCC tissues was negatively associated with tumor size, pathological grade, clinical stage and tumor recurrence. Representative results of selected TCC and corresponding normal urothelium tissues are shown in Fig. 2.

Loss of PTPN12 activity increases the proliferation of TCC cells. A pcDEF3 expression vector, which contained the full length cDNA for PTPN12, was stably transfected into four TCC cell lines (253J, T24, TCCSUP and RT112). PTPN12 expression was also reduced using RNAi technology. PTPN12 expression in all transfected TCC cell lines was confirmed by RT-PCR; PTPN12 mRNA expression was significantly increased by the PTPN12 vector insert and decreased by RNAi (Fig. 3A). The effect of PTPN12 on the proliferation of TCC cells in vitro was assessed by a WST-1 assay. TCC cells expressing high PTPN12, exhibited significantly decreased proliferative ability compared with the control cells. By contrast TCC cells expressing low PTPN12 expression exhibited a high proliferative ability compared with 
the control group (Fig. 3B). In addition, the effect of PTPN12 on the proliferation of TCC cells was confirmed in vivo using xenografts in BALB/C nude mice (Fig. 3C). These findings suggested that decreased PTPN12 expression may be involved in the proliferation of human TCC.

Loss of PTPN12 activity increases the adhesion, migration and invasion of TCC cells. Recurrence, an important cause of mortality in patients with malignancy, is a complex multistep process, which includes changes in cell adhesion, migration and invasion (19). The effects of PTPN12 on the adhesion, migration and invasion of TCC cells were evaluated in the present study. The expression of PTPN12 in two TCC cell lines was decreased using RNAi, and PTPN12 expression was increased by transfection with the pcDEF3 vector, which contained the full length cDNA of PTPN12. PTPN12 expression was evaluated using RT-PCR (Fig. 3A). As shown in Fig. 4, TCC cells with a low expression of PTPN12 due to siRNA treatment, exhibited significantly increased levels of adhesion (Fig. 4A), migration (Fig. 4B) and invasion (Fig. 4C) compared with untreated controls. By contrast, TCC cells with a high expression of PTPN12, exhibited reduced levels of adhesion (Fig. 4A), migration (Fig. 4B) and invasion (Fig. 4C) compared with control cells. These findings suggested that decreased PTPN12 expression may enhance the adhesion, migration and invasion of TCC cells, and that PTPN12 is involved in the recurrence of TCC.

\section{Discussion}

Bladder urothelial carcinoma is the ninth most common malignancy in humans, and the incidence is nearly 3-fold higher in males than females (20). TCC is a life-long disease, as the rate of recurrence is high and thus, patients require regular out-patient check-ups for the rest of their lives. Certain patients may undergo repeated surgery and postoperative bladder perfusion chemotherapy. Therefore, routine intravesical instillation with different chemotherapeutic agents is performed in order to prevent recurrence of TCC following transurethral resection, and radical cystectomy is usually recommended for patients with muscle-invasive and high-frequency recurrent disease (21). Although TCC is sensitive to chemotherapy, no agents are currently available, which specifically eliminate recurrence $(22,23)$. The identification of novel molecular mechanisms and therapeutic targets for TCC are required.

Recently, PTPN12 has been hypothesized to be a tumor suppressor gene. Loss of PTPN12 phosphatase activity has been reported to cause cellular transformation in mammary epithelial cells and aberrant acinar morphogenesis in human breast cancer (12). In recent years, decreased expression of PTPN12 has been observed in human breast cancer, and this is associated with tumor progression and a poor prognosis (11). PTPN12 expression is also significantly decreased in hepatocellular carcinoma (HCC) tissues compared with normal liver tissues, and PTPN12 is an independent predictor of reduced cancer-specific and recurrence-free survival in patients with HCC (24). Although these studies have demonstrated that PTPN12 is involved in the development of human tumors, the function of PTPN12 in TCC is unclear, and the effect of PTPN12 on the proliferation of TCC cells requires further elucidation. To the best of our knowledge, the current study is the first to focus on PTPN12 expression in human TCC. The present study analyzed the expression of PTPN12 in human TCC, and the results of immunohistochemical analysis demonstrated that its expression is significantly decreased in TCC tissues compared with normal urothelium tissues. In addition, PTPN12 expression was significantly associated with tumor size, pathological grade, clinical stage and tumor recurrence. PTPN12 expression in TCC was also measured using RT-qPCR and western blotting, and the results of these analyses were in accordance with those obtained using immunohistochemistry. The effect of PTPN12 on the proliferation of TCC cells was also measured. The results showed that reduced expression of PTPN12 significantly increased the proliferative capacity of TCC cells in vitro. Similar results were obtained using xenografts in BALB/C nude mice. Therefore, the current study demonstrated that PTPN12 is an important gene in carcinogenesis and is involved in the progression of TCC.

Although a number of studies have been conducted in order to better predict recurrence in patients with TCC, the mechanism of tumor recurrence remains unknown (25-27). The PTPN12 protein is an important regulator of cell adhesion, motility and invasion as a result of its inhibition of, and interaction, with multiple oncogenic tyrosine kinases (28). Therefore, it is hypothesized that PTPN12 may be involved in the recurrence of TCC. A previous study reported that the recurrence of TCC is associated with spread by intraluminal seeding (19), and tumor recurrence is known to be a complex multistep biological behavior that includes cell adhesion, migration and invasion (29). Therefore, the effect of PTPN12 on the adhesion, migration and invasion of TCC cells was investigated in the current study. It was demonstrated that reduced expression of PTPN12 enhances the adhesion, migration and invasion properties of TCC cells. Furthermore, PTPN12 expression was significantly decreased in the group with recurrent TCC, compared with the primary TCC group. These finding suggest that PTPN12 may enhance the adhesion, migration and invasion of TCC cells, thereby facilitating the recurrence of TCC.

It was recently reported that PTPN12 acts as a suppressor of epithelial cell motility by regulating the assembly of adherent junctions and the activity of Rho GTPase in colon cancer (14). Another study concluded that PTPN12 acts as a negative regulator of cell motility via the regulation of FAK activity by HER2 (10). Promoter hypermethylation is known to be a key molecular mechanism, which leads to the gene silencing of tumor suppressor genes. A recent study reported that hypermethylation in the promoter $\mathrm{CpG}$ island is frequently detected, and is related to low PTPN12 expression, which suggests that hypermethylation in the promoter $\mathrm{CpG}$ island may be an important mechanism regulating the expression of PTPN12 (11). Although the correlation between PTPN12 expression and progression in tumors has been analyzed in various studies, the molecular mechanisms underlying the effects of PTPN12 in TCC remain elusive and require further investigation.

In conclusion, the present results showed that PTPN12 expression is downregulated in human TCC, and that decreased expression of PTPN12 may lead to the progression and recurrence of this disease. It may be that patients with a low level of PTPN12 expression are at increased risk of the 
progression and recurrence of TCC, and PTPN12 may be a useful predictive factor for TCC recurrence. The current study also suggested that restoring PTPN12 expression may be a novel therapeutic strategy, and that the molecular mechanisms underlying the effects of PTPN12 in TCC require further elucidation.

\section{Acknowledgements}

This study is supported by grant of China State Scholarship Fund (grant no. 201408220020) and Jilin Province 125 Scientific and Technological Research Project and YanBian University Science and Technology Development Item (grant no. 2014039).

\section{References}

1. Ploeg M, Aben KK and Kiemeney LA: The present and future burden of urinary bladder cancer in the world. World J Urol 279: 289-293, 2009.

2. Siegel R, Ma J, Zou Z and Jemal A: Cancer statistics, 2014. CA Cancer J Clin 64: 9-29, 2014.

3. Jacobs BL, Lee CT and Montie JE: Bladder cancer in 2010: How far have we come? CA Cancer J Clin 60: 244-272, 2010.

4. Shariat SF, Karakiewicz PI, Palapattu GS, et al: Outcomes of radical cystectomy for transitional cell carcinoma of the bladder: A contemporary series from the Bladder Cancer Research Consortium. J Urol 176: 2414-2422, 2006.

5. Morgan TM and Clark PE: Bladder cancer. Curr Opin Oncol 22 242-249, 2010

6. Kumar V, Abbas AK and Fausto N: 8th Philadelephia: Elsevier; Pathologic Basis of Disease 976-980, 2010.

7. Hsu JL, Huang SY, Chow NH and Chen SH: Stable-isotope dimethyl labeling for quantitative proteomics. Anal Chem 75: 6843-6852, 2003.

8. Yarden Y and Sliwkowski MX: Untangling the ErbB signalling network. Nat Rev Mol Cell Biol 2: 127-137, 2001.

9. Hunter T: Tyrosine phosphorylation: Thirty years and counting. Curr Opin Cell Biol 21: 140-146, 2009.

10. Villa-Moruzzi E: Tyrosine phosphatases in the HER2-directed motility of ovarian cancer cells: Involvement of PTPN12, ERK5 and FAK. Anal Cell Pathol (Amst) 34: 101-112, 2011.

11. Xunyi Y,Zhentao Y, Dandan J and Funian L: Clinicopathological significance of PTPN12 expression in human breast cancer. Braz J Med Biol Res 45: 1334-1340, 2012.

12. Sun T, Aceto N, Meerbrey KL, et al: Activation of multiple proto-oncogenic tyrosine kinases in breast cancer via loss of the PTPN12 phosphatase. Cell 144: 703-718, 2011.

13. Cao X, Li Y, Luo RZ, He LR, et al: Tyrosine-protein phosphatase nonreceptor type 12 is a novel prognostic biomarker for esophageal squamous cell carcinoma. Ann Thorac Surg 93: 1674-1680, 2012.

14. Espejo R, Rengifo-Cam W, Schaller MD, Evers BM and Sastry SK: PTPPEST controls motility, adherens junction assembly and Rho GTPase activity in colon cancer cells. Am J Physiol Cell Physiol 299: C454-C463, 2010.
15. Zheng Y, Yang W, Xia Y, et al: Ras-induced and extracellular signal-regulated kinase 1 and 2 phosphorylation-dependent isomerization of protein tyrosine phosphatase (PTP)-PEST by PIN1 promotes FAK dephosphorylation by PTP-PEST. Mol Cell Biol 31: 4258-4269, 2011.

16. Davidson D, Shi X, Zhong MC, Rhee I and Veillette A: The phosphatase PTP-PEST promotes secondary $\mathrm{T}$ cell responses by dephosphorylating the protein tyrosine kinase Pyk2. Immunity 33: 167-180, 2010.

17. Streit S, Ruhe JE, Knyazev P, et al: PTP-PEST phosphatase variations in human cancer. Cancer Genet Cytogenet 170: 48-53, 2006.

18. Kapur U, Antic T, Venkataraman G, Durazo-Arvizu R, Quek MM, Flanigan RC and Wojcik EM: Validation of World Health Organization/International Society of Urologic Pathology 2004 classification schema for bladder urothelial carcinomas using quantitative nuclear morphometry: Identification of predictive features using bootstrap method. Urology 70: 1028-1033, 2007.

19. Streit S, Ruhe JE, Knyazev P, et al: PTP-PEST phosphatase variations in human cancer. Cancer Genet Cytogenet 170: 48-53, 2006.

20. Lerner SP: Bladder cancer clinical trials. Urol Oncol 23: 275-279, 2005.

21. Mitsumori K, Tsuchiya N, Habuchi T, et al: Early and large-dose intravesical instillation of epirubicin to prevent superficial bladder carcinoma recurrence after transurethral resection. BJU Int 94: 317-321, 2004.

22. Sylvester RJ, Oosterlinck W and van der Meijden AP: A single immediate postoperative instillation of chemotherapy decreases the risk of recurrence in patients with stage Ta T1 bladder cancer: A meta-analysis of published results of randomized clinical trials. J Urol 171: 2186-2190, 2004.

23. Brausi M, Collette L and Kurth K: Variability in the recurrence rate at first follow up cystoscopy after TUR in stage Ta T1 transitional cell carcinoma of the bladder: A combined analysis of seven EORTC studies. Eur Urol 41: 523-531, 2002.

24. Luo RZ, Cai PQ, Li M, et al: Decreased expression of PTPN12 correlates with tumor recurrence and poor survival of patients with hepatocellular carcinoma. PLoS One 9: e85592, 2014.

25. Ajili F, Darouiche A, Chebil M and Boubaker S: The efficiency of the EORTC scoring system for the prediction of recurrence and progression of non-muscle-invasive bladder cancer treated by bacillus Calmette-Guerin immunotherapy. Ultrastruct Pathol 37: 249-253, 2013.

26. Mares J, Szakacsova M, Soukup V, Duskova J, Horinek A and Babjuk M: Prediction of recurrence in low and intermediate risk non-muscle invasive bladder cancer by real-time quantitative PCR analysis: cDNA microarray results. Neoplasma 60: 295-301, 2013.

27. Xu T, Zhu Z, Zhang X, Wang X, Zhong S, Zhang M and Shen Z: Predicting recurrence and progression in chinese patients with nonmuscle-invasive bladder cancer using EORTC and CUETO scoring models. Urology 82: 387-393, 2013.

28. Hafner C, Knuechel R, Zanardo L, et al: Evidence for oligoclonality and tumor spread by intraluminal seeding in multifocal urothelial carcinomas of the upper and lower urinary tract. Oncogene 20: 4910-4015, 2001

29. Shang D, Liu Y, Yang P, Chen Y and Tian Y: TGFBI-promoted adhesion, migration and invasion of human renal cell carcinoma depends on inactivation of von Hippel-Lindau tumor suppressor. Urology 79: e1-e7, 2012. 